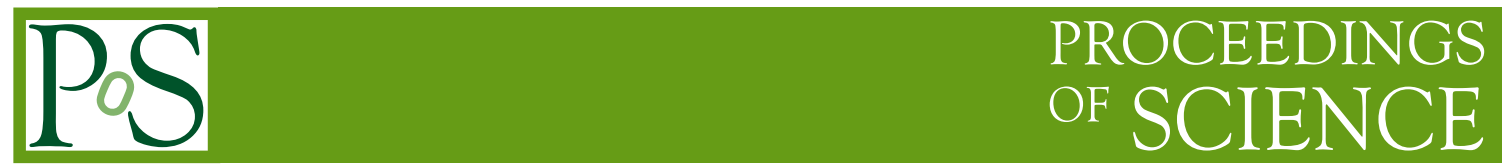

\title{
Maximal Unitarity at Two Loops
}

\section{Henrik Johansson}

Theory Group, Physics Department, CERN

CH-1211 Geneva 23, Switzerland

\section{David A. Kosower*}

Institut de Physique Théorique, CEA-Saclay,

F-91191 Gif-sur-Yvette cedex, France

\section{Kasper J. Larsen}

NIKHEF, Science Park 105,

NL-1098 XG Amsterdam, The Netherlands

We discuss the extension of the maximal-unitarity method to two loops, focusing on the example of the planar double box. Maximal cuts are reinterpreted as contour integrals, with the choice of contour fixed by the requirement that integrals of total derivatives vanish on it. The resulting formulæ, like their one-loop counterparts, can be applied either analytically or numerically.

Loops and Legs in Quantum Field Theory - 11th DESY Workshop on Elementary Particle Physics, April 15-20, 2012

Wernigerode, Germany

\footnotetext{
*Speaker.
} 


\section{Introduction}

Amplitudes are the basic building blocks for physics predictions in QCD. Predictions of differential cross sections are essential to controlling backgrounds to new physics at the Large Hadron Collider (LHC). Because of their strong dependence on the unphysical renormalization and factorization scales, leading-order (LO) predictions are not quantitatively reliable. Next-to-leading order (NLO) calculations give the first quantitative predictions of processes involving QCD. NLO calculations require one-loop amplitudes, in addition to other ingredients. Recent years have seen a revolution in our ability to calculate these amplitudes, thanks to maximal unitarity [1,2] and other developments [4] such as the Ossola-Papadopoulos-Pittau [3] decomposition.

For some processes, such as $g g \rightarrow W^{+} W^{-}$and $g g \rightarrow Z Z$, the tree-level amplitude vanishes, and accordingly the one-loop amplitude furnishes only an LO prediction. Such subprocesses are nominally higher order in the strong coupling, of $\mathscr{O}\left(\alpha_{s}^{2}\right)$, compared to $\mathscr{O}\left(\alpha_{s}^{0}\right)$ for the basic $q \bar{q} \rightarrow W^{+} W^{-}, \mathrm{ZZ}$ subprocesses. This is however partly compensated by the much larger gluon densities, so that they merit computation. To compute these subprocesses to NLO, one needs twoloop amplitudes.

Two-loop amplitudes are also needed for NNLO calculations, which in turn will be needed for future precision physics at the LHC. Such calculations will also be useful in providing honest uncertainty estimates for existing NLO predictions.

\section{On-Shell Methods}

Traditional Feynman-diagrammatic methods suffer from an explosion in the number of diagrams, and an even greater explosion in the number of terms, as the number of external legs or the number of loops increases. Yet many results for amplitudes, especially in the $\mathscr{N}=4$ supersymmetric gauge theory, are extremely compact; and all known loop results in gauge theories are vastly more compact than would be suggested by the number of diagrams. This reflects the vast redundancy present in Feynman diagrams, due to explicit handling of non-physical states, and the resulting gauge dependence of intermediate quantities. On-shell methods use only information from physical, on-shell, states to compute amplitudes, thereby avoiding throughout the computation of gauge-variant quantities which must cancel at the end. This makes calculations simpler and made possible new NLO calculations at high multiplicity, such as those of $W, Z+4$ jets and $W+5$ jets [5].

On-shell methods make use of general properties of amplitudes to derive tools for computations: tree-level factorization leads to the Britto-Cachazo-Feng-Witten on-shell recursion relations for tree amplitudes [6]; the unitarity of the $S$-matrix gives rise to the unitarity [7] and generalizedunitarity methods; and the presence of an underlying field theory allows for a representation in terms of an integral basis. The formalism can be summarized in the following equation,

$$
\text { Amplitude }=\sum_{j \in \text { Basis }} c_{j} \operatorname{Int}_{j}+\text { Rational },
$$

where the sum is taken over a basis of integrals, and the coefficients $c_{j}$ as well as the remaining rational terms are rational functions of spinor variables. For analytic calculations, having a basis 


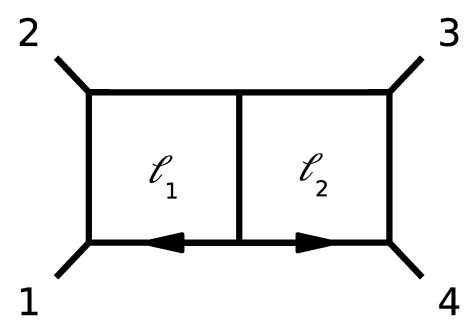

Figure 1: The double-box integral

of linearly-independent integrals simplifies calculations but is not strictly essential. For numerical calculations, it is essential.

\section{Integral Basis}

At one loop, the basis for computations in massless gauge theories consists of box, triangle, and bubble integrals, where all internal lines are massless, and external legs may be massive or massless. In general, we must distinguish between two different notions of basis: a " $D$-dimensional basis," which keeps terms to all orders in the dimensional regulator $\varepsilon$, and a "regulated fourdimensional basis," which keeps only terms through $\mathscr{O}\left(\varepsilon^{0}\right)$. Because some integrals are linearly independent only at $\mathscr{O}(\varepsilon)$, the latter basis is more compact. We will implicitly be using this latter basis in these Proceedings. The planar part of the basis at two loops will contain integrals with up to eight propagators [8]. Beyond one loop, some basis integrals will necessarily contain irreducible numerators, numerators which cannot be written as linear combinations of inverse propagators. For the double boxes we consider here and shown in fig. 1, for example, there are two basis integrals when all external legs are massless, or when one external leg is massive.

\section{Maximal Unitarity}

In the basic unitarity method at one loop, we sew together two tree amplitudes with a phasespace integral, and promote the positive-energy on-shell delta functions to off-shell propagators. The resulting object will yield an integral containing the correct contribution to the target amplitude in the channel in which we have performed the sewing. We must still reduce the resulting integral symbolically in order to separate the contributions from different basis integrals, and find their respective coefficients. Finally, we must merge contributions from all channels. This sewing procedure inverts the procedure of cutting a one-loop amplitude, in which we replace a pair of propagators surrounding the given channel by positive-energy delta functions. This isolates all integrals containing those two propagators. There are of course many such integrals: various boxes and triangles, and a bubble integral as well.

In order to isolate a smaller number of integrals, we must cut more propagators. This is possible; indeed at one loop, we have four degrees of freedom in the loop momentum (ignoring the $(-2 \varepsilon)$-dimensional components), and so we can imagine cutting four propagators at once [2]. There is, however, a subtlety involved. We might imagine replacing the four propagators in the box 
integral,

$$
\int \frac{d^{4-2 \varepsilon} \ell}{(2 \pi)^{4-2 \varepsilon}} \frac{1}{\ell^{2}\left(\ell-k_{1}\right)^{2}\left(\ell-K_{12}\right)^{2}\left(\ell+k_{4}\right)^{2}},
$$

by positive-energy delta functions,

$$
\int \frac{d^{4-2 \varepsilon} \ell}{(2 \pi)^{4-2 \varepsilon}} \delta^{(+)}\left(\ell^{2}\right) \delta^{(+)}\left(\left(\ell-k_{1}\right)^{2}\right) \delta^{(+)}\left(\left(\ell-K_{12}\right)^{2}\right) \delta^{(+)}\left(\left(\ell+k_{4}\right)^{2}\right) .
$$

These delta functions instruct us to solve the simultaneous equations,

$$
\ell^{2}=0, \quad-2 \ell \cdot k_{1}+k_{1}^{2}=0, \quad-2 \ell \cdot k_{2}+K_{12}^{2}-k_{1}^{2}=0, \quad 2 \ell \cdot k_{4}+k_{4}^{2}=0,
$$

which are linear combinations of the delta-function arguments. Let us examine the special case when legs 1, 2, and 4 are massless; we can then solve the first, second, and last equations by setting,

$$
\ell^{\mu}=\frac{\xi}{2}\left\langle 1^{-}|\mu| 4^{-}\right\rangle
$$

and then solve for $\xi$,

$$
\xi=-\frac{[12]}{[24]},
$$

using the third equation in eq. (4.3). Similarly, we see that there is a second solution,

$$
\ell^{\mu}=-\frac{\langle 12\rangle}{2\langle 24\rangle}\left\langle 4^{-}|\mu| 1^{-}\right\rangle .
$$

The subtlety arises from the fact that for generic external momenta, these solutions are complex. The domain of the delta functions, on the other hand, is real; taken literally, the delta functions would yield zero! Cutting both sides of eq. (2.1) would then give us the equation $0=0$, which is true but not very useful. This is the same issue that arises in straightforward interpretations of delta functions in the connected picture for twistor-string amplitudes [9].

To find a solution to this subtlety, we may note [10] that contour integration behaves very much like integration over a delta function,

$$
\oint_{C\left(z_{0}\right)} d z \frac{\operatorname{Poly}_{1}(z)}{\operatorname{Poly}_{2}(z)-a}=\frac{\operatorname{Poly}_{1}\left(z_{0}\right)}{\operatorname{Poly}_{2}^{\prime}\left(z_{0}\right)}
$$

where $z_{0}$ is defined by the equation $\operatorname{Poly}_{2}\left(z_{0}\right)=a$ (with multiciplicity one). We could define the desired delta function as follows,

$$
\int d z \operatorname{Poly}_{1}(z) \delta\left(\operatorname{Poly}_{2}(z)-a\right) \equiv \oint_{C\left(z_{0}\right)} d z \frac{\operatorname{Poly}_{1}(z)}{\operatorname{Poly}_{2}(z)-a} .
$$

There is one significant difference from ordinary delta function integration: there is no absolute value around the derivative in the denominator on the right-hand side of eq. (4.7), so that the result remains an analytic function.

That is, we must reinterpret cutting propagators as contour replacement: instead of replacing the propagators by delta functions, we replace the original contours of integration, along the real axes of the now-complexified loop momenta $\ell^{\mu}$, by contours surrounding the global poles, that is 


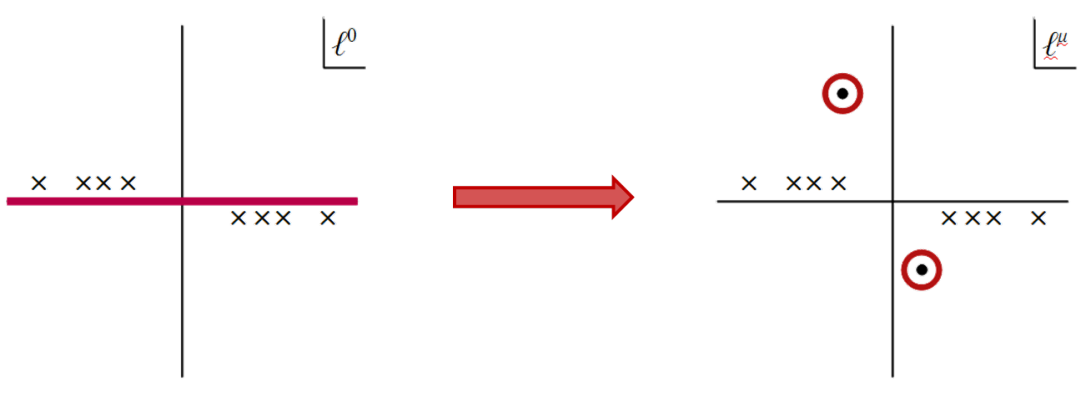

Figure 2: Cutting as contour replacement

the simultaneous solutions to eqs. (4.3), in $\mathbb{C}^{4}$. The contour in the case of the one-loop box is a product of four circles, that is a four-torus $T^{4}$. The replacement is illustrated schematically in fig. 2 , with contours $\mathscr{C}_{1,2}$ encircling the two global poles. We stress that this is not a contour deformation leaving the value of the integral unchanged; it is a replacement, changing the value of the integral and ultimately allows us to derive an equation for the coefficient of the one-loop box.

This reinterpretation raises two new problems, however. We have to choose $T^{4}$ contours surrounding two global poles, around which we could wind an arbitrary number of times (even a fractional number of times). How should we choose the contour? Also, replacing the contour by such an arbitrary winding can break integral identities. For example, the identity,

$$
0=\int \frac{d^{4-2 \varepsilon} \ell}{(2 \pi)^{4-2 \varepsilon}} \frac{\varepsilon\left(\ell, k_{1}, k_{2}, k_{4}\right)}{\ell^{2}\left(\ell-k_{1}\right)^{2}\left(\ell-K_{12}\right)^{2}\left(\ell+k_{4}\right)^{2}},
$$

is spoiled if we choose the contour to encircle just one of the global poles.

Remarkably, these two problems cancel each other out. If we take a general contour, $\mathscr{C}=$ $a_{1} \mathscr{C}_{1}+a_{2} \mathscr{C}_{2}$, we find that the integral in eq. (4.9) takes the value,

$$
\left(a_{1}-a_{2}\right) f\left(k_{1}, k_{2}, k_{4}\right)
$$

so that it will still vanish if $a_{1}=a_{2}$. This fixes the contour up to an overall irrelevant constant which will cancel out of eq. (2.1). Applying the cutting via contour replacement to our basic equation (2.1), we can derive a formula for the coefficient of the corresponding one-loop box. The resulting equation is the same as the one obtained by Britto, Cachazo, and Feng (BCF) [2].

Let us now apply these ideas to the double-box integral (fig. 1). In the same way that this derivation can be seen as a generalization to two loops of the formalisms of BCF and Forde [11], recent work on two-loop integrands by Mastrolia, Mirabella, Ossola, and Peraro [12] and by Badger, Frellesvig, and Zhang [13,14] can be seen as the two-loop generalization of the OssolaPapadopoulos-Pittau construction [3]. The maximal cut in the double box involves cutting seven propagators. Each solution has one continuous degree of freedom $z$. The number of distinct solutions depends on the number and configuration of external masses. When all four external legs are massless; when one external leg is massive; when two diagonally-opposite legs (for example, legs 1 and 3) are massive; or when two long-edge legs (for example, legs 1 and 4) are massive, there are six solutions. We will call these configurations 'class (c)'. When two short-edge legs (for example, legs 1 and 2) are massive, or when three legs are massive, there are four solutions. We will label 
these configurations 'class (b)'. (This classification is explained in ref. [15].) Performing the corresponding contour integrals gives rise to a Jacobian factor (the analog for the maximal cut in the double box of the Poly ${ }_{2}^{\prime}\left(z_{0}\right)$ factor in eq. (4.7)). Here, the resulting Jacobian is a function of $z$; it has poles in $z$, so that we can choose a contour for the $z$ integration as well, thereby obtaining a global pole. After identifying different parametrizations of the same global pole arising from different solutions to the heptacut equations, and taking into account poles that arise in the loop momenta $\ell_{1,2}$ as well as in the Jacobian, we see that there are always eight global poles, independent of the number and configuration of external masses [15].

Unlike the number of global poles, the number of independent basis integrals does depend on the number and configuration of external masses. In class (c), there are two basis integrals; in class (b), there are three. We would like to construct independent "projectors", which give formulæ for the coefficients of each of these basis integrals. Each projector will be a linear combination of contours around the global poles. How should we choose them?

We again impose the requirement, analogous to eq. (4.9), that all vanishing loop integrals continue to vanish on the chosen contours. As at one loop, there are vanishing integrals where a Levi-Civita tensor is inserted into the numerator of the scalar integral. There are five possible integrals, which give rise to four independent constraints on the contours. In addition, there are integration-by-parts (IBP) identities [16, 17] which give 20 linear relations in class (c) between the 22 integrals with different powers of the two irreducible numerators $\ell_{1} \cdot k_{4}$ and $\ell_{2} \cdot k_{1}$. Not all of the resulting constraints on the contours are independent; in class (c), we find two independent constraints, while in class (b) we have only 19 IBP equations, which reduce to a single constraint. We work here to leading order in $\varepsilon$, leaving higher-order terms in the coefficients to future work. The constraints leave us with two independent contours for the two master integrals in class (c), and three independent contours for the three master integrals in class (b). We can obtain a projector for any given integral by imposing the further constraint that the other integrals vanish on the contour, and that it reproduces the integral itself with unit coefficient.

The formulæ for the coefficients of double boxes take the following form,

$$
c=i \sum_{j=1}^{8} a_{j} \oint_{T^{8}\left(\mathscr{G}_{j}\right)} d^{8} v_{a, b} J_{\oint, j} \sum_{\substack{\text { particles } \\ \text { helicities }}} \prod_{p=1}^{6} A_{p}^{(0)}\left(v_{a, b}\right),
$$

where $A^{(0)}$ are tree-level amplitudes in the gauge theory, and the $a_{j}$ are weights (or winding numbers) for the different global poles. For example, for the one-mass double box (with $m_{1}^{2} \neq 0$ ) the weights $a_{j}$ for the two basis integrals, $I[1]$ and $I\left[\ell_{1} \cdot k_{4}\right]$, are,

$$
\left(a_{j}\right)=\frac{1}{4}(1,1,1,1,0,0,1,1),
$$

and

$$
\left(a_{j}\right)=\frac{m_{1}^{2}-s_{12}}{2 s_{12} s_{14}}(1,1,1,1,-2,-2,3,3),
$$

respectively. The solutions and global poles are given in terms of a parametrization of the loop momenta given in ref. [18], where the reader may also find complete formulæ for the solutions, global poles, and projectors for classes (b) and (c).

This work is supported by the European Research Council under Advanced Investigator Grant ERC-AdG-228301. 


\section{References}

[1] Z. Bern, L. J. Dixon and D. A. Kosower, Nucl. Phys. B 513, 3 (1998) [hep-ph/9708239].

[2] R. Britto, F. Cachazo and B. Feng, Nucl. Phys. B 725, 275 (2005) [hep-th/0412103].

[3] G. Ossola, C. G. Papadopoulos and R. Pittau, Nucl. Phys. B 763, 147 (2007) [hep-ph/0609007].

[4] C. Anastasiou, R. Britto, B. Feng, Z. Kunszt and P. Mastrolia, Phys. Lett. B 645, 213 (2007) [hep-ph/0609191]; JHEP 0703, 111 (2007) [hep-ph/0612277]; W. T. Giele, Z. Kunszt and K. Melnikov, JHEP 0804, 049 (2008) [arXiv:0801.2237 [hep-ph]]; C. F. Berger and D. Forde, Ann. Rev. Nucl. Part. Sci. 60, 181 (2010) [arXiv:0912.3534 [hep-ph]]; R. K. Ellis, W. T. Giele and Z. Kunszt, JHEP 0803, 003 (2008) [0708.2398 [hep-ph]]; C. F. Berger, Z. Bern, L. J. Dixon, F. Febres Cordero, D. Forde, H. Ita, D. A. Kosower and D. Maître, Phys. Rev. D 78, 036003 (2008) [0803.4180 [hep-ph]]; G. Ossola, C. G. Papadopoulos and R. Pittau, JHEP 0803, 042 (2008) [arXiv:0711.3596 [hep-ph]]; P. Mastrolia, G. Ossola, C. G. Papadopoulos and R. Pittau, JHEP 0806, 030 (2008) [arXiv:0803.3964 [hep-ph]]; W. T. Giele and G. Zanderighi, JHEP 0806, 038 (2008) [arXiv:0805.2152 [hep-ph]]; R. K. Ellis, W. T. Giele, Z. Kunszt, K. Melnikov and G. Zanderighi, JHEP 0901, 012 (2009) [arXiv:0810.2762 [hep-ph]].

[5] C. F. Berger, Z. Bern, L. J. Dixon, F. Febres Cordero, D. Forde, T. Gleisberg, H. Ita, D. A. Kosower, and D. Maître, Phys. Rev. Lett. 106, 092001 (2011) [arXiv:1009.2338 [hep-ph]]; H. Ita, Z. Bern, L. J. Dixon, F. Febres Cordero, D. A. Kosower and D. Maître, Phys. Rev. D 85, 031501 (2012) [arXiv:1108.2229 [hep-ph]]; Z. Bern, G. Diana, L. J. Dixon, F. Febres Cordero, D. Forde, T. Gleisberg, S. Hoeche, H. Ita, D. A. Kosower, D. Maître, and K. Ozeren, these proceedings.

[6] R. Britto, F. Cachazo, B. Feng and E. Witten, Phys. Rev. Lett. 94, 181602 (2005) [hep-th/0501052].

[7] Z. Bern, L. J. Dixon, D. C. Dunbar and D. A. Kosower, Nucl. Phys. B 425, 217 (1994) [hep-ph/9403226]; Nucl. Phys. B 435, 59 (1995) [hep-ph/9409265].

[8] J. Gluza, K. Kajda and D. A. Kosower, Phys. Rev. D 83, 045012 (2011) [arXiv:1009.0472 [hep-th]].

[9] R. Roiban, M. Spradlin and A. Volovich, Phys. Rev. D 70, 026009 (2004) [hep-th/0403190].

[10] C. Vergu, Phys. Rev. D 75, 025028 (2007) [hep-th/0612250].

[11] D. Forde, Phys. Rev. D 75, 125019 (2007) [0704.1835 [hep-ph]].

[12] P. Mastrolia and G. Ossola, JHEP 1111, 014 (2011) [arXiv:1107.6041 [hep-ph]]; P. Mastrolia, E. Mirabella, G. Ossola and T. Peraro, arXiv:1205.7087 [hep-ph]; P. Mastrolia, E. Mirabella, G. Ossola and T. Peraro, arXiv:1209.4319 [hep-ph].

[13] S. Badger, H. Frellesvig and Y. Zhang, JHEP 1204, 055 (2012) [arXiv:1202.2019 [hep-ph]]; JHEP 1208, 065 (2012) [arXiv:1207.2976 [hep-ph]].

[14] Y. Zhang, JHEP 1209, 042 (2012) [arXiv:1205.5707 [hep-ph]].

[15] S. Caron-Huot and K. J. Larsen, JHEP 1210, 026 (2012) [arXiv:1205.0801 [hep-ph]].

[16] F. V. Tkachov, Phys. Lett. B 100, 65 (1981); K. G. Chetyrkin and F. V. Tkachov, Nucl. Phys. B 192, 159 (1981).

[17] S. Laporta, Phys. Lett. B 504, 188 (2001) [hep-ph/0102032]; S. Laporta, Int. J. Mod. Phys. A 15, 5087 (2000) [hep-ph/0102033].

[18] H. Johansson, D. A. Kosower and K. J. Larsen, arXiv:1208.1754 [hep-th]. 